\title{
Prevalence of cerebral edema among diabetic ketoacidosis patients
}

\author{
Paula González Pannia, M.D. ${ }^{a}$, Romina Balboa, M.D. ${ }^{a}$, Rocío Navarro, M.D. ${ }^{a}$, \\ Milva F. Nocita, M.D. ${ }^{a}$, Mabel Ferraro, M.D. ${ }^{a}$ and Carla Mannucci, M.D. ${ }^{a}$
}

\begin{abstract}
Introduction. Cerebral edema (CE) is the most severe complication of diabetic ketoacidosis (DKA) in children. There is no accurate knowledge of CE pathogenesis and its onset has been related to intravenous rehydration therapy during the initial treatment.

Objectives. To estimate the prevalence of $\mathrm{CE}$ among DKA patients treated at Hospital General de Niños Pedro de Elizalde with intravenous rehydration and analyze potential risk factors for the development of CE.

Materials and methods. Cross-sectional prevalence study and exploratory analysis to compare clinical and laboratory characteristics between patients with and without CE. Patients aged 1-18 years hospitalized with the diagnosis of DKA between January $1^{\text {st }}, 2005$ and December $31^{\text {st }}, 2014$ were included.

Results. A total of 693 DKA events from 561 medical records were analyzed. Ten patients had evidence of CE $(1.44 \%$; $95 \%$ confidence interval: 0.8-2.6). Patients with $\mathrm{CE}$ had higher serum urea levels $(p<0.001)$, lower carbon dioxide pressure $(p<0.001)$, and lower serum sodium levels $(p<0.001)$ than those without CE. Conclusion. The prevalence of CE among DKA patients was $1.44 \%$, smaller than that reported in our country $(1.8 \%)$. The risk factors at admission associated with CE development were high serum urea levels, hyponatremia, and hypocapnia.

Key words: cerebral edema, diabetic ketoacidosis, diabetes mellitus, child.
\end{abstract}

http: / / dx.doi.org/10.5546/ aap.2020.eng.332

a. Hospital General de Niños Pedro de Elizalde, Autonomous City of Buenos Aires, Argentina.

E-mail address:

Paula González Pannia,

M.D.:

pau.gp@hotmail.com

Funding:

None.

Conflict of interest:

None.

Received: 7-26-2019.

Accepted: 1-2-2020.

\section{INTRODUCTION}

Diabetic ketoacidosis (DKA) is a severe complication of diabetes and has a high morbidity and mortality. It is a common cause of hospitalization and the leading cause of death among pediatric diabetes patients. ${ }^{1}$

Cerebral edema (CE) is the most severe complication of DKA in children. Its prevalence in Argentina is $1.8 \%$ according to a multicenter study conducted by the Argentine Society of Diabetes, ${ }^{2}$ higher than that reported in studies that found it to range between $0.5 \%$ and $1 \% . .^{3,4}$ Symptomatic CE is the most severe manifestation of a pathophysiological phenomenon common to all types of ketoacidosis; $95 \%$ of cases occur in patients younger than 20 years. ${ }^{3}$

There is no accurate knowledge of CE pathogenesis. Some investigators have attributed it to a cellular edema caused by rapid plasma osmolality changes during intravenous rehydration therapy. ${ }^{4}$ Sub-clinical CE may be present at the beginning of any pediatric DKA case, regardless of management. ${ }^{5,6}$

There are currently different methods for the hydration of patients diagnosed with DKA. At Hospital General de Niños Pedro de Elizalde (HGNPE), the treatment standard establishes an initial expansion with saline solution at a rate of $20 \mathrm{~mL} / \mathrm{kg}$ of body weight. Then, patients receive intravenous rehydration at a rate of $4200 \mathrm{~mL}$ per $\mathrm{m}^{2}$ of body surface area in the first 24 hours, with half of fluids administered in the first 6 hours and the rest, in the following 18 hours. ${ }^{78}$

The objective of this study was to estimate the prevalence of CE and potential risk factors associated with its development among DKA patients managed at HGNPE. 


\section{MATERIALS AND METHODS Design}

This was a cross-sectional prevalence study followed by an exploratory analysis to compare clinical and laboratory characteristics between patients with and without CE. The analysis was developed for all patients with CE and a selected sample of patients without $C E$.

\section{Population}

The medical records of patients aged 1-18 years hospitalized at HGNPE with the diagnosis of DKA between January 1 $1^{\text {st }}, 2005$ and December $31^{\text {st }}, 2014$ were included. DKA was defined as a condition meeting the following biochemical criteria: blood glucose $>200 \mathrm{mg} /$ $\mathrm{dL}, \mathrm{pH}<7.30$ and / or bicarbonate $<15 \mathrm{mmol} / \mathrm{L}$ with glycosuria and urine ketones. The baseline hydration protocol as per the HGNPE standards ${ }^{7}$ remained the same during the entire study period. Patients who had received hydration therapy other than the one indicated by the HGNPE standards and those with a known disease or treatment that may have altered electrolyte or acid-base balance were excluded.

\section{Sample size}

To meet the primary objective and expecting a $1.5 \% \pm 0.5 \%$ prevalence, with a $99 \%$ confidence level, in a population with 693 DKA events in the study period, the sample size was estimated to be, at least, 589 DKA events.

Given that this is a low-prevalence event, to explore the risk factors associated with the development of $\mathrm{CE}$, the total number of $\mathrm{CE}$ events $(n=10)$ were included and 15 events without CE were randomly selected for each DKA event with $\mathrm{CE}(\mathrm{n}=150) .{ }^{9}$ For selection, medical records were ordered from older to newer and were assigned a consecutive number. The 150 required cases were then selected using a table of random numbers. If one was not eligible, the immediately following one was selected.

\section{Outcome measure selection and definition}

Outcome variable. Cerebral edema yes / no (categorical outcome measure). Cerebral edema secondary to DKA was defined based on the presence of two major clinical criteria or one major and two minor criteria (major criteria: altered sensorium, bradycardia, urinary incontinence; minor criteria: vomiting, headache, lethargy, diastolic blood pressure [DBP] above $90 \mathrm{mmHg}$, age younger than 5 years). ${ }^{10}$
Predictive outcome measures. Patient age: expressed in years (continuous outcome measure). Form of disease onset: diabetes debut yes/no (categorical outcome measure). Baseline biochemical parameters (all included patients had venous blood lab tests done before any therapeutic intervention provided at the HGNPE):

- Serum urea in $\mathrm{mg} / \mathrm{dL}$ as continuous outcome measure.

- Serum sodium in $\mathrm{mEq} / \mathrm{L}$ as continuous outcome measure.

- Glucose-corrected blood sodium in $\mathrm{mEq} / \mathrm{L}$ : it was based on the following formula: actual sodium in $\mathrm{mEq} / \mathrm{L}-[$ (blood glucose in $\mathrm{mg} /$ $\mathrm{dL}-100) \times 1.6 / 100)]$. This was analyzed as a continuous outcome measure.

- Partial pressure of carbon dioxide $\left(\mathrm{pCO}_{2}\right)$ in mmHg as continuous outcome measure.

- $\mathrm{pH}$ in arbitrary units as continuous outcome measure.

- Plasma bicarbonate in $\mathrm{mEq} / \mathrm{L}$ as continuous outcome measure (measured based on an estimation of $\mathrm{pH}$ and $\mathrm{pCO}_{2}$ ).

Control outcome measure. Sex, female/male (categorical outcome measure).

Data collection and statistical analysis. The medical records of patients hospitalized due to DKA in the study period were used as analysis units. All medical records used included all the data required for analysis. The routine registry sheet included major and minor criteria for the diagnosis of CE. Data were collected and stored in a standardized, specifically-designed data collection sheet. Data were completely dissociated from patients' identity.

CE prevalence was described based on CE cases out of all DKA cases observed in the study period, with a $95 \%$ confidence interval (CI). The sample was divided into two groups to explore risk factors: patients with $\mathrm{CE}$ and without $\mathrm{CE}$. Categorical outcome measures were described as frequency of distribution and percentage, whereas quantitative outcome measures were expressed as median (range) because of their nonparametric distribution.

A univariate analysis was performed. Categorical outcome measures were compared using Fisher's exact test and expressed as an odds ratio and its corresponding $95 \%$ CI. Quantitative outcome measures were compared with the Wilcoxon test. The statistical analysis was done with the Statistix 7.0 software; a value of $p<0.05$ was considered statistically significant. 
Ethical considerations. In accordance with the rules governing research in the setting of facilities dependent on the Government of the Autonomous City of Buenos Aires, study approval was requested before the hospital's Ethics Committee and Research and Teaching Committee. All data were anonymized, so we requested the Ethics Committee for authorization to waive the informed consent. The study was registered before the Research Ethics Committee of the Ministry of Health of the Autonomous City of Buenos Aires (347/14).

\section{RESULTS}

Prevalence of cerebral edema. A total of 719 DKA cases were admitted to HGNPE in the study period. Twenty-six of them were excluded because they were initially treated at a different center (Figure 1). A total of 693 DKA events were analyzed in 561 patients. CE was evidenced in 10 of them, which accounted for a CE prevalence in our hospital of $1.44 \%$ (95\% CI: 0.8-2.6).

Baseline biochemical outcome measures. To study potential risk factors, 160 DKA events were analyzed: 150 in the group without CE and 10 in the CE group. Among all patients, 33 corresponded to disease onset, while the rest had a previous diagnosis of type 1 diabetes mellitus. No association was observed between disease onset and the risk for CE. In relation to studied biochemical outcome measures, CE patients had high serum urea levels and more commonly hyponatremia and hypocapnia (Table 1).

\section{DISCUSSION}

In this study, the prevalence of CE was observed to be $1.44 \%$ (95\% CI: 0.79-2.6). Among patients who developed $\mathrm{CE}$, the baseline lab tests showed a higher blood urea level and a lower blood sodium level and $\mathrm{pCO}_{2}$ than those without CE.

In our sample, CE prevalence was $1.44 \%$, lower than what had been previously reported in an Argentine multicenter study (1.8\%). That study analyzed patients from different children's hospitals across the country and it was observed that each center followed their own treatment protocols. For all DKA events, except for those managed in our hospital,

FIGURE 1. Flowchart of the study

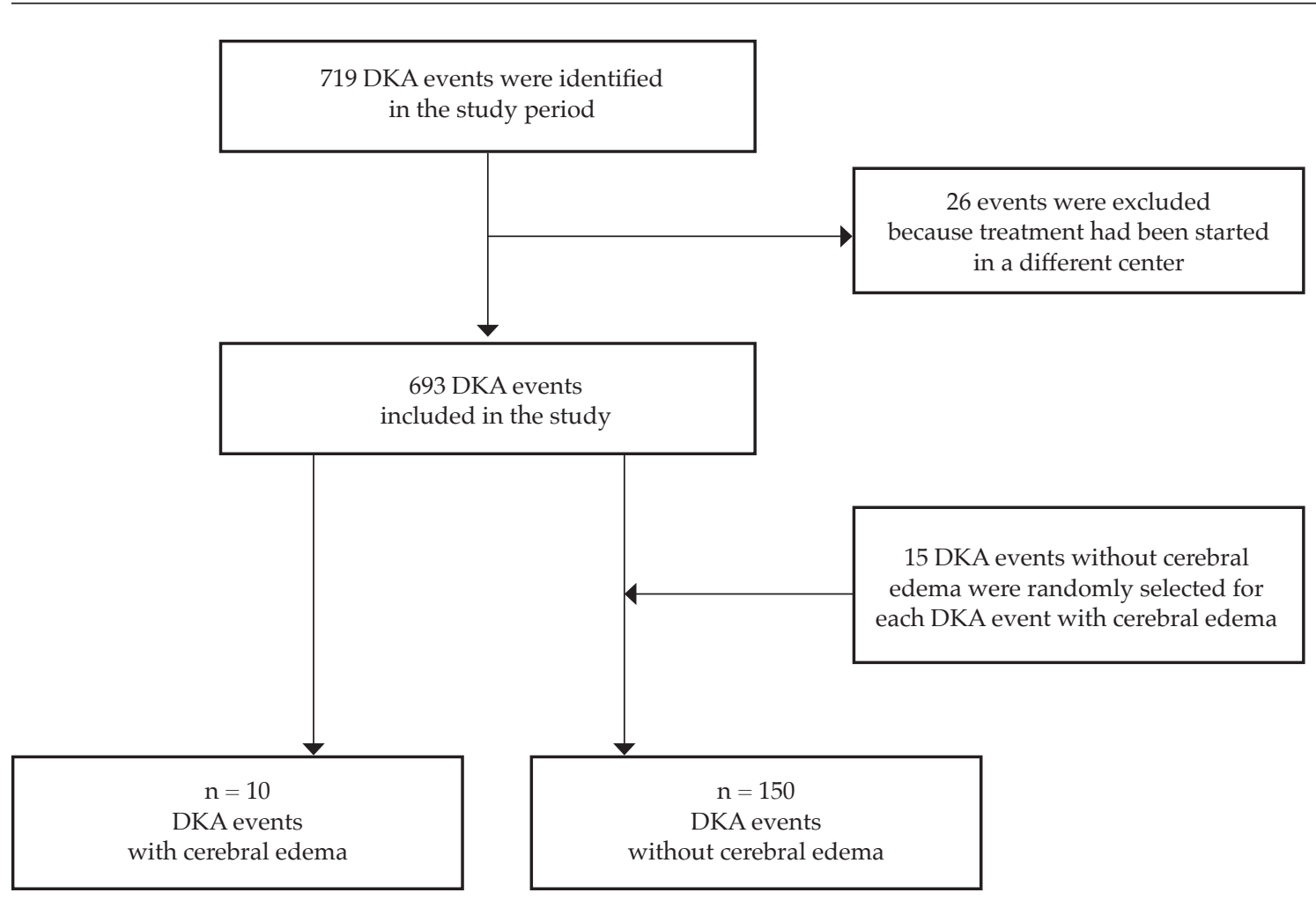

DKA: diabetic ketoacidosis. 
patients were administered a constant volume over the first 24 hours. The fact that the CE prevalence described in this study was lower than that described for Argentina reinforced the concept that the rate of fluid infusion may not be associated with development of CE. ${ }^{2}$

CE pathophysiology in DKA is still controversial. In the past, several factors had been described to cause $C E$, including osmotic edema, which may be caused by the administration of large fluid volumes in the first hours of treatment. ${ }^{11}$ However, although all DKA patients have a fluctuating osmolality, less than $2 \%$ develop CE. ${ }^{11}$ More recent data have suggested that CE in DKA patients is associated with cerebral ischemia due to hypoperfusion. ${ }^{4}$ Hypocapnia caused by metabolic acidosis may lead to cerebral vasoconstriction, which, together with dehydration, causes a reduced blood flow to the brain, thus generating cerebral ischemia, brain injury, and cytotoxic edema. Rehydration could cause a reperfusion injury, post-ischemic hyperemia, and vasogenic edema. ${ }^{4}$

In 2017, Glaser et al. compared the changes in blood flow distribution in different brain regions among DKA patients using magnetic resonance imaging (MRI) at admission, 3 hours and 6 hours after recovery. They described an increased blood flow in some regions of the brain and a reduction in others, thus suggesting concurrent vasogenic and cytotoxic cerebral edema in the same patient. ${ }^{13}$

In a recently published multicenter clinical trial, patients were divided into four groups depending on the treatment they received $(0.9 \%$ or $0.45 \%$ sodium chloride and slow or fast administration rate). No statistically significant difference was observed in the rate of CE among the patients who received the different treatments. These findings would emphasize the lack of a causative association between the rate of fluid administration and the development of clinically significant cerebral edema. ${ }^{12}$ In the same regard, up to $19 \%$ of patients with CE have it at the time of admission. ${ }^{5}$ This suggests that factors previous to treatment, as well as any individual susceptibility, may play a major role in the development of $\mathrm{CE}$, regardless of treatmentassociated factors.

In relation to the risk factors associated with $\mathrm{CE}$, in our study, CE patients had higher baseline blood urea values $(>40 \mathrm{mmol} / \mathrm{L})$ and a lower baseline $\mathrm{pCO}_{2}(<20 \mathrm{mmHg})$ than patients without CE. They also had lower blood sodium and glucose-corrected blood sodium levels than the group without CE. These data are similar to those published in other studies. ${ }^{4,10} \mathrm{~A}$ high suspicion index for CE should be applied in these patients because an early identification and a timely treatment significantly reduce mortality. ${ }^{14}$

The median age of patients with and without edema was 11 and 13 years, respectively. Consistent with other authors, no significant differences were observed between the patients of both groups. ${ }^{4,5}$

We found a lack of association between disease onset and CE. Although, consistent with previous studies, ${ }^{4,5}$ there is a lack of association among these outcome measures, other authors have described that patients with disease debut have a higher risk for $\mathrm{CE} .{ }^{10}$ There is still not enough evidence to draw a conclusion on this topic.

The study has limitations due to its retrospective nature. However, it was possible

TABLE 1. Characteristics of patients before treatment initiation

\begin{tabular}{|c|c|c|c|c|}
\hline & $\begin{array}{c}\mathrm{CE} \\
(\mathrm{n}=10)\end{array}$ & $\begin{array}{l}\text { Without CE } \\
(\mathrm{n}=150)\end{array}$ & OR $(95 \% \mathrm{CI})$ & $p$ \\
\hline Age (years) & $11.5(3.0-18.0)$ & $13.1(2.0-18.8)$ & & 0.25 \\
\hline Sex (male/female) & $7 / 3$ & $60 / 90$ & $3.5(0.9-13.9)$ & 0.09 \\
\hline Debut (yes/no) & $3 / 7$ & $25 / 125$ & $2.1(0.5-8.9)$ & 0.38 \\
\hline Blood sodium (mEq/L) & $134(132-141)$ & $140(126-155)$ & & $<0.001$ \\
\hline Corrected blood sodium $(\mathrm{mEq} / \mathrm{L})$ & $129(125-135)$ & $134(119-149)$ & & $<0.001$ \\
\hline Blood urea $(\mathrm{mg} / \mathrm{dL})$ & $61(47-89)$ & $37(15-102)$ & & $<0.001$ \\
\hline $\mathrm{CO}_{2}$ pressure $(\mathrm{mmHg})$ & $20.3(15.0-28.4)$ & $27.2(10.5-52.0)$ & & $<0.001$ \\
\hline $\mathrm{pH}$ (arbitrary units) & $7.17(6.94-7.24)$ & $7.17(6.67-7.4)$ & & 0.52 \\
\hline Bicarbonate (mEq/L) & $7.0(4.1-12.0)$ & $9.3(2.2-17.0)$ & & 0.06 \\
\hline
\end{tabular}

Categorical outcome measures were described as proportions with their corresponding ORs (95\% CI).

Continuous outcome measures were reported as median (range). A value of $\mathrm{p}<0.05$ was considered significant.

$\mathrm{CE}$ : cerebral edema; $\mathrm{OR}$ : odds ratio; $\mathrm{CI}$ : confidence interval; $\mathrm{CO}_{2}$ : carbon dioxide. 
to prevent missing data because, in compliance with the hospital's regulations, a standardized form was completed for all DKA patients, which included the data used in this study. In addition, although certain risk factors were identified, given the low prevalence of this event, the number of patients was not enough to identify the cut-off values of predictor outcome measures for CE development.

\section{CONCLUSION}

The prevalence of CE among DKA patients was $1.44 \%$, and the risk factors at admision for its development were high serum urea, hypocapnia and hyponatremia.

\section{REFERENCES}

1. Piva JP, Czepielewski M, García PC, Machado D. Current perspectives for treating children with diabeticketoacidosis. J Pediatr (Rio J). 2007; 83(5 Suppl):S119-27.

2. Ferraro M, Ozuna B, Mazza C, Ramos O, et al. Estudio multicéntrico de cetoacidosis diabética en servicios pediátricos de Argentina. Rev Soc Argent Diabetes. 2009; 43(3)127-36.

3. Muir A, Quisling R, Yang M, Rosembloom A. Cerebral edema in childhood diabetic ketoacidosis: natural history, radiographic findings, and early identification. Diabetes Care. 2004; 27(7):1541-6.

4. Glaser N, BarnettP, McCaslin I, Nelson D, et al. Risk factors for cerebral edema in children with diabetic ketoacidosis. The Pediatric Emergency Medicine Collaborative Research
Committee of the American Academy of Pediatrics. N Engl J Med. 2001; 344(4):264-9.

5. Lawrence S, Cummings E, Gaboury I, Daneman D. Population-based study of incidence and risk factors for cerebral edema in pediatric diabetic ketoacidosis. J Pediatr. 2005; 146(5):688-92.

6. Krane E, Rockoff M, Wallman J, Wolfsdorf J. Subclinical Brain Swelling in Children during Treatment of Diabetic Ketoacidosis. N Engl J Med. 1985; 312(18):1147-51.

7. Ramos O, Ferraro M. Cetoacidosis diabética. In:Voyer Luis E. Criterios de diagnóstico y tratamiento en Pediatría. 2da ed. Buenos Aires: Journal; 2012.Pages.95-9.

8. FerreiraJ,PenazziM, TaboadaM,FunesS, etal.Comparación de dos sistemas de aporte de soluciones hidroelectrolíticas para el manejo de la cetoacidosis diabética: ensayo clínico controlado y aleatorizado en niños. Rev Fac Cienc Med Córdoba. 2015; 72(2):93-9.

9. Fleiss JL, Levin B. Sample size determination in studies with matched pairs. J Clin Epidemiol. 1988; 41(8):727-30.

10. Wolfjdor JI, Allgrove J, Craig M,EdgeJA, et al. ISPAD clinical practice consensus guidelines 2014. Diabetic ketoacidosis and hyperglycemic hyperosmolar state. Pediatr Diabetes. 2014; 15(Suppl 20):154-79.

11. Long B, Koyfman A. Emergency medicine myths: cerebral edema in pediatric diabetic ketoacidosis and intravenous fluids. J Emerg Med. 2017; 53(5):212-21.

12. Kuppermann N, Ghetti S, Schunk J, Stoner M, et al. Clinical trial of fluid infusion rates for pediatric diabetes ketoacidosis. N Engl J Med. 2018; 378(24):2275-87.

13. Glaser N, Wootton-Gorges SL, Kim I, Tancredi D, et al. Regional brain water content and distribution during diabetic ketoacidosis. J Pediatr. 2017; 180:170-6.

15. Edge JA, Jakes RW, Roy Y, Hawkins M, et al. The UK casecontrol study of cerebral edema complicating diabetic ketoacidosis in children. Diabetologia. 2006; 49(9):2002-9. 